\title{
Rapid energization of radiation belt electrons by nonlinear wave trapping
}

\author{
Y. Katoh ${ }^{1,2}$, Y. Omura ${ }^{1}$, and D. Summers ${ }^{1,3}$ \\ ${ }^{1}$ Research Institute for Sustainable Humanosphere, Kyoto University, Gokasho, Uji, Kyoto 611-0011, Japan \\ ${ }^{2}$ Planetary Plasma and Atmospheric Research Center, Graduate School of Science, Tohoku Univ., Sendai 980-8578, Japan \\ ${ }^{3}$ Dept. of Mathematics and Statistics, Memorial University of Newfoundland, St. John's, Newfoundland, A1C 5S7 Canada
}

Received: 7 October 2008 - Accepted: 29 October 2008 - Published: 6 November 2008

\begin{abstract}
We show that nonlinear wave trapping plays a significant role in both the generation of whistler-mode chorus emissions and the acceleration of radiation belt electrons to relativistic energies. We have performed particle simulations that successfully reproduce the generation of chorus emissions with rising tones. During this generation process we find that a fraction of resonant electrons are energized very efficiently by special forms of nonlinear wave trapping called relativistic turning acceleration (RTA) and ultra-relativistic acceleration (URA). Particle energization by nonlinear wave trapping is a universal acceleration mechanism that can be effective in space and cosmic plasmas that contain a magnetic mirror geometry.
\end{abstract}

Keywords. Magnetospheric physics (Energetic particles, trapped) - Space plasma physics (Nonlinear phenomena; Wave-particle interactions)

\section{Introduction}

Whistler-mode chorus waves are electromagnetic emissions that have been observed by spacecraft in the magnetospheres of Earth (Tsurutani and Smith, 1974; Meredith et al., 2001; Santolik et al., 2004), Jupiter (Scarf et al., 1979; Coroniti et al., 1980), Saturn (Gurnett et al., 1981; Hospodarsky et al., 2008), and Uranus (Gurnett et al., 1986), with frequencies in the range $0.05-0.8 \Omega_{e}$, where $\Omega_{e}$ is the local electron gyrofrequency. At Earth chorus emissions have also been recorded on the ground (Smith et al., 2004). Chorus waves comprise discrete emissions of short duration $\left(<10^{-1} \mathrm{~s}\right)$, with frequency typically increasing in time ("rising tones"), and, when connected to an audio amplifier, sound like birdsong at dawn. Theoretical and modeling studies (Summers et al., 1998, 2002; Roth et al., 1999; Summers and Ma,

Correspondence to: Y. Katoh

(yuto@pparc.geophys.tohoku.ac.jp)
2000; Albert, 2002; Miyoshi et al., 2003; Varotsou et al., 2005; Omura and Summers, 2006) and observational evidence (Meredith et al., 2001, 2003) have established electron acceleration by gyroresonant wave-particle interaction with chorus waves as a key mechanism for generating relativistic electrons in the Earth's outer radiation belt during geomagnetically disturbed periods. After nearly four decades of chorus wave observations and numerical studies, only recently have computer simulations been performed that successfully model the generation of chorus emissions with a rising tone (Katoh and Omura, 2007a; Omura et al., 2008). Here we show that in the simulation of chorus generation, some resonant electrons are rapidly energized by the processes of relativistic turning acceleration (RTA) (Omura et al., 2007; Katoh and Omura, 2007b) and ultra-relativistic acceleration (URA) (Summers and Omura, 2007). RTA and URA are particular forms of nonlinear wave trapping of resonant electrons by coherent whistler-mode waves and constitute viable mechanisms for the generation of relativistic electrons in the radiation belts of magnetized planets (Furuya et al., 2008). The computer simulations reveal that nonlinear wave growth due to the rising tone is the key ingredient in the generation of chorus waves.

\section{Generation mechanism of chorus emissions}

Necessary conditions for chorus generation in the equatorial region of the Earth's outer radiation belt include the injection of energetic seed electrons from the outer magnetosphere during magnetospheric disturbances known as substorms; see Fig. 1a. Recent theory and simulations (Omura et al., 2008) show that chorus emissions can be generated in a dipole magnetic field by seed electrons with a large temperature anisotropy in the energy range $\sim 10$ s of $\mathrm{keV}$. Whistler-mode waves propagating parallel to the magnetic field grow from electromagnetic thermal noise near the 


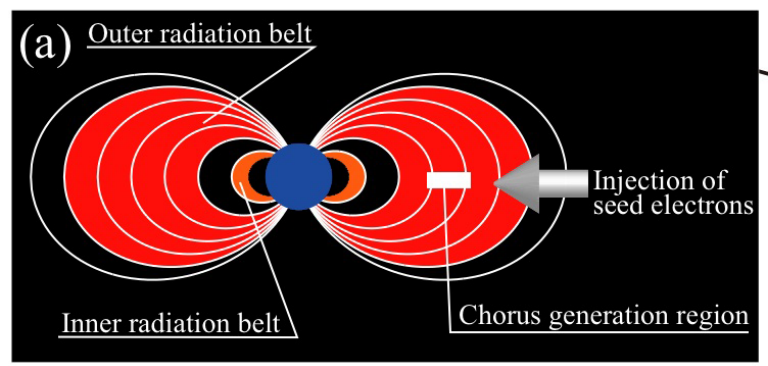

(b)
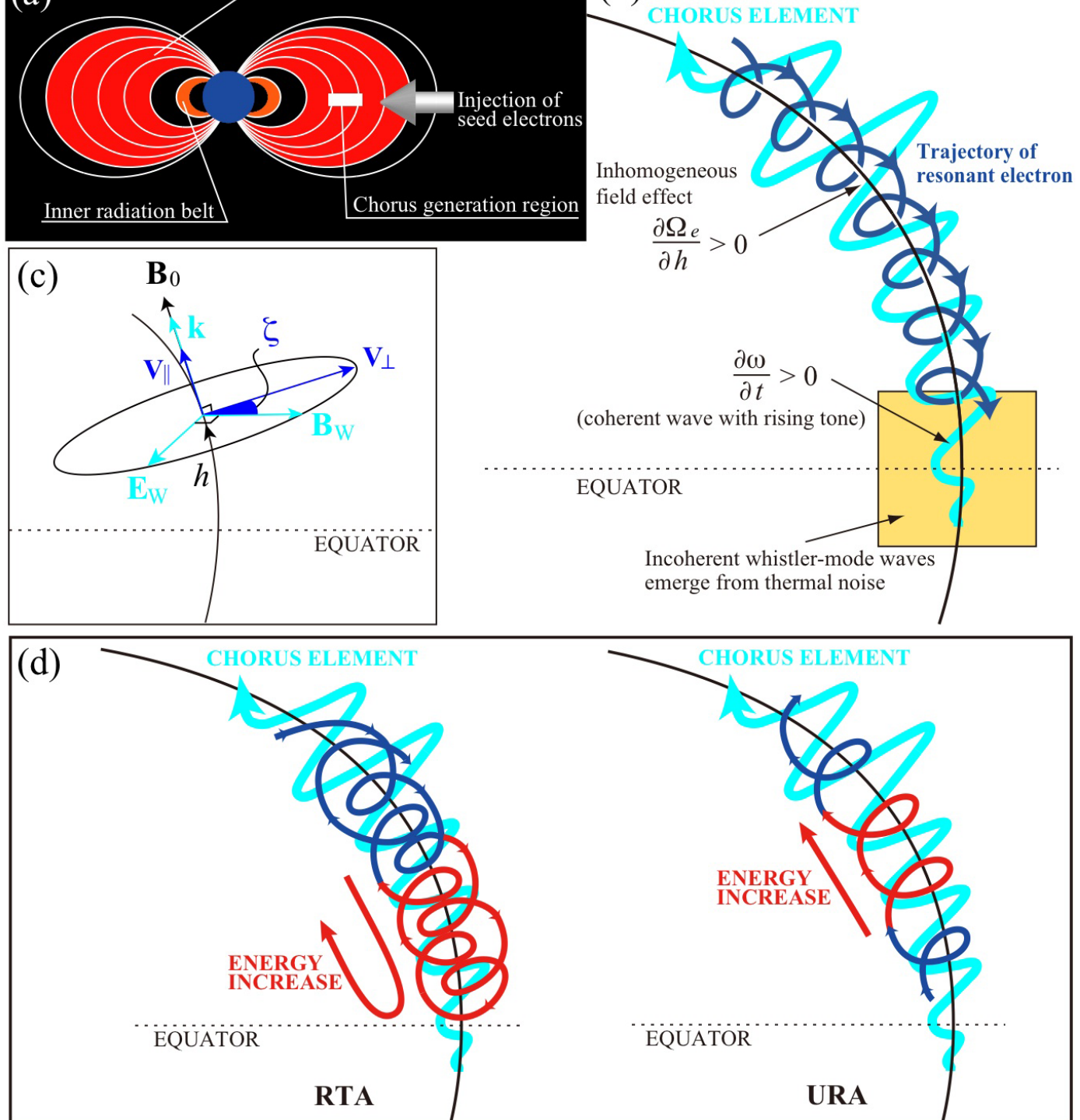

Fig. 1. (a) Earth's radiation belts; (b) Chorus wave generation near the equator; (c) Vector geometry of a whistler-mode wave in a dipole magnetic field, and velocity components of a resonant electron; (d) The relativistic turning acceleration (RTA) and ultra-relativistic acceleration (URA) mechanisms operating near the equator.

equator, receiving energy from energetic electrons that satisfy the cyclotron resonance condition,

$\omega-k v_{\|}=\frac{\Omega_{e}(h)}{\gamma}$,

where $\omega$ and $k$ are the wave frequency and wavenumber, respectively. The left-hand side of Eq. (1) is the Dopplershifted frequency of the wave, and the right-hand side is the relativistic cyclotron frequency given by the cyclotron frequency $\Omega_{e}(h)$ of non-relativistic electrons divided by the Lorenz factor $\gamma=\left[1-\left(v_{\|}^{2}+v_{\perp}^{2}\right) / c^{2}\right]^{-1 / 2} ; v_{\|}$and $v_{\perp}$ are the components of electron velocity parallel and perpendic- ular to the background magnetic field, respectively, and $c$ is the speed of light. The variable $h$ is the distance along the magnetic field measured from the equator. A band of incoherent whistler-mode waves in the frequency range 0.1$0.5 \Omega_{e}(0)$ forms near the equator, as predicted by linear theory (Kennel and Petschek, 1966). Nonlinear wave growth (Omura et al., 2008) takes place for a coherent wave component with a phase variation corresponding to an increasing frequency or rising tone. That is, from the enhanced incoherent wave spectrum, a coherent wave packet with increasing frequency $(\partial \omega / \partial t>0)$ corresponding to maximum nonlinear wave growth emerges as a seed of a chorus element, as illustrated in Fig. 1b. We have reproduced the gradual formation 

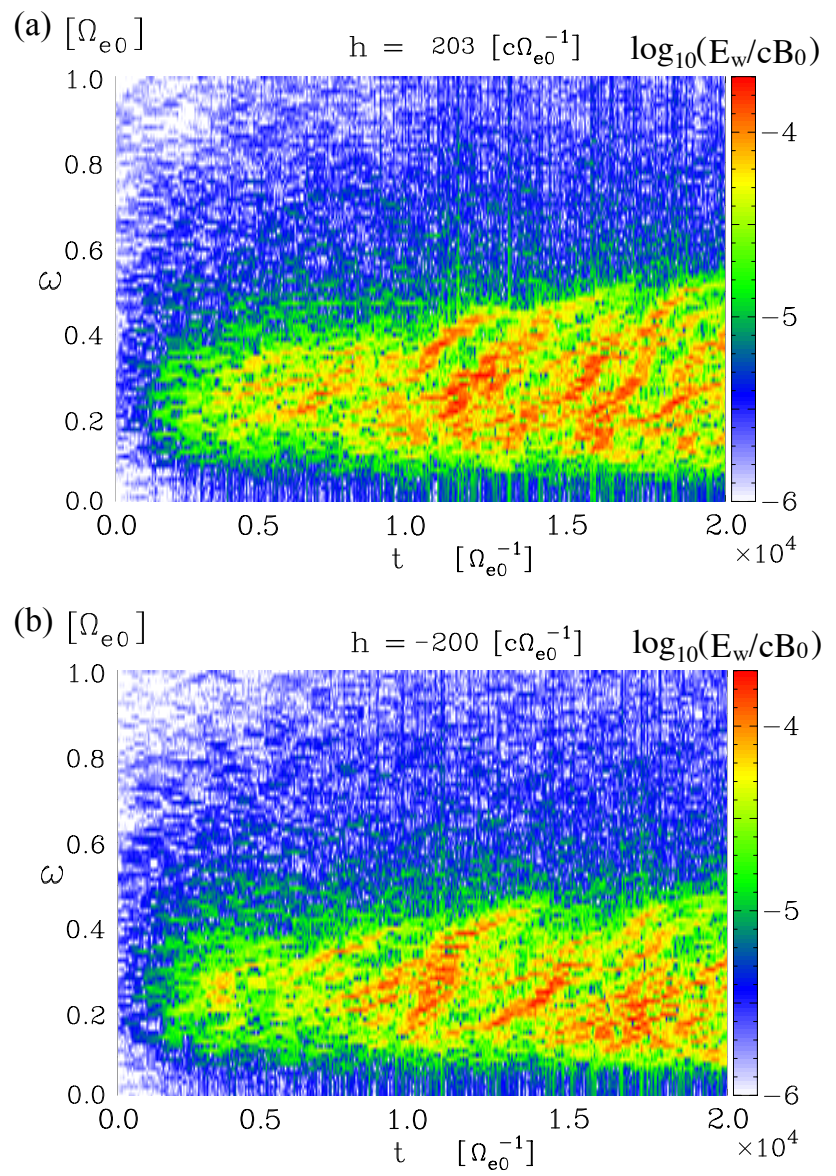

Fig. 2. Spectrograms generated in the Northern Hemisphere (top) and the Southern Hemisphere (bottom). The vertical axis represents wave frequency $\omega ; \Omega_{e 0}$ is the electron gyrofrequency at the equator. Several chorus emissions are generated in both hemispheres in the simulation. These waves propagate in the poleward directions and interact nonlinearly with counter-streaming energetic electrons. The majority of the resonant electrons are untrapped and lose energy slightly, thereby contributing to chorus wave generation. Selected electrons are trapped by the generated chorus and undergo dramatic acceleration by the RTA/URA processes.

of coherent whistler-mode waves by means of a large-scale computer simulation. The dynamical frequency spectra of the simulated chorus emissions are shown in Fig. 2.

Once the seed of a chorus element is formed near the equator, it propagates away from the equator and undergoes further nonlinear growth due to both the rising tone and the increasing gradient of the magnetic field; this is illustrated in the wave amplitude profile generated in the simulation and plotted in Fig. 3a. The nonlinear growth is sustained so long as sufficient energetic electrons exist with velocities close to the resonance velocity $v_{\|}=V_{R}$ specified by the cyclotron resonance condition (1). The dynamics of the resonant electrons are described by a modified pendulum equation derived from the second-order resonance condition for stable trapping of resonant electrons and given by
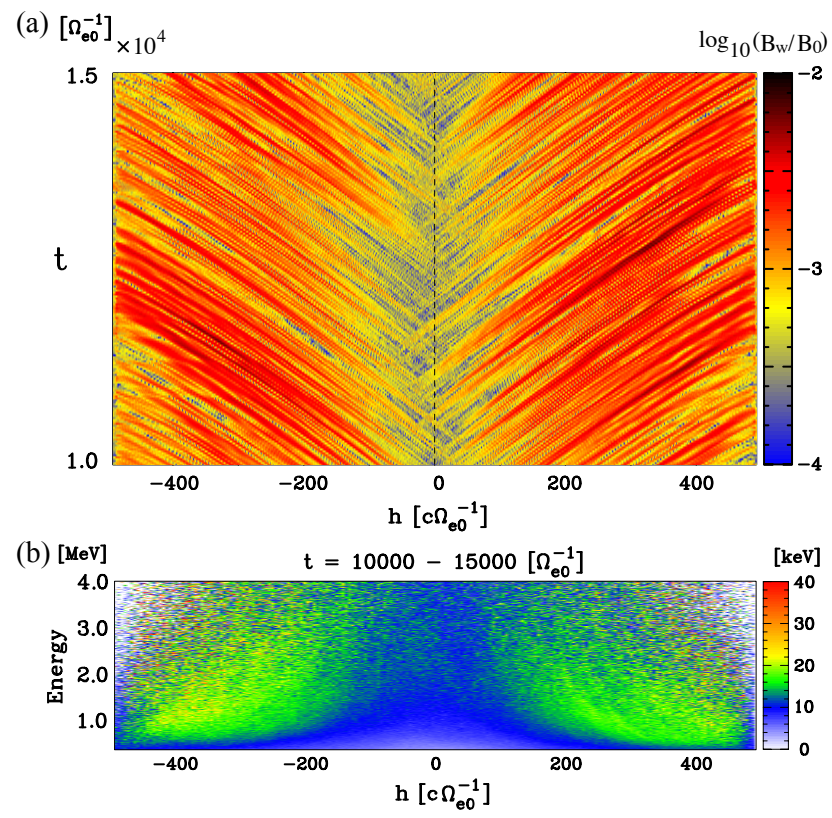

Fig. 3. The top panel shows the spatial profile of the amplitude of the transverse wave magnetic field and its time evolution. Modes with longer wavelengths than chorus emissions are eliminated. Wave packets of whistler-mode waves are successively generated near the magnetic equator, their amplitudes increasing as they propagate into both hemispheres. The bottom panel shows the spatial distribution of the energy increase of accelerated electrons, as a function of kinetic energy and position at $t=10000 \Omega_{e 0}^{-1}$. The efficiency of the acceleration depends on both the wave amplitude of the chorus emissions and the variation of the resonant velocity $V_{R}$. The variation of $V_{R}$ is due to both the spatial inhomogeneity of the background magnetic field and the wave frequency of the chorus emissions.

$\frac{d^{2} \zeta}{d t^{2}}=\frac{\omega_{t}^{2} \delta^{2}}{\gamma}(\sin \zeta+S)$,

where $\omega_{t}=\sqrt{k v_{\perp} \Omega_{W}}$ is the trapping frequency and $\delta^{2}=1-\omega^{2} / c^{2} k^{2}$. The parameter $\Omega_{W}$ is defined by $\Omega_{W}=e B_{W} / m_{0}$ where $B_{W}, e$ and $m_{0}$ are the amplitude of the wave magnetic field, the unit charge and the rest mass of an electron. The variable $\zeta$ is the phase angle between the perpendicular velocity $\boldsymbol{v}_{\perp}$ of a resonant electron and the wave magnetic field $\boldsymbol{B}_{W}$ in the plane transverse to the dipole magnetic field; see Fig. 1c. The variation of the phase, and likewise of the center of the trapping potential, is controlled by the inhomogeneity ratio (Omura et al., 2008),

$$
\begin{aligned}
S= & -\frac{1}{\omega_{t}^{2} \delta^{2}}\left\{\gamma\left(1-\frac{V_{R}}{V_{g}}\right)^{2} \frac{\partial \omega}{\partial t}\right. \\
& \left.+\left[\frac{k \gamma v_{\perp}^{2}}{2 \Omega_{e}}-\left(1+\frac{\delta^{2}}{2} \frac{\Omega_{e}-\gamma \omega}{\Omega_{e}-\omega}\right) V_{R}\right] \frac{\partial \Omega_{e}}{\partial h}\right\} .
\end{aligned}
$$

The first and second terms in the curly brackets represent the effects of the rising tone and the spatial inhomegeneity, 


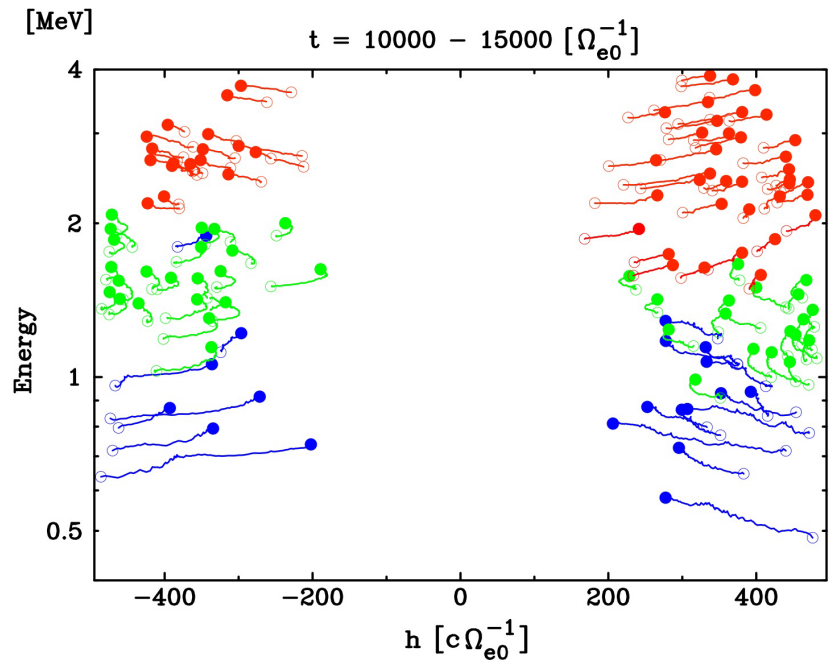

Fig. 4. Examples of trajectories of accelerated electrons during the time interval, $t=10000 \Omega_{e 0}^{-1}-15000 \Omega_{e 0}^{-1}$. Blue, green and red curves show trajectories of electrons accelerated through the normal trapping, RTA and URA processes, respectively. Open and filled circles respectively indicate trapping and detrapping points on each trajectory. In the energy range corresponding to $\gamma<\Omega_{e} / \omega$, trapped electrons move toward the magnetic equator during their acceleration. For a trapped electron satisfying the RTA turning condition $\gamma=\Omega_{e} / \omega$, the resonance velocity becomes zero due to the relativistic effect and eventually changes sign, thereby resulting in the turning motion. Since the gyro-frequency of highly relativistic electrons is smaller than the frequency of chorus waves, trapped electrons then move in the direction of the chorus emissions that are moving away from the equator. Highly relativistic electrons trapped by multiple chorus elements can undergo multiple URA interactions.

respectively. When $S \sim-0.4$, an electron hole is produced in the velocity phase space giving rise to a maximum resonant current that induces the nonlinear wave growth. Since $\omega_{t}^{2}$ is proportional to the wave amplitude $B_{W}$, the contribution of $\partial \omega / \partial t$ to the quantity $|S|$ decreases as the wave amplitude increases. On the other hand, the increasing inhomogeneity of the magnetic field $\left(\partial \Omega_{e} / \partial h\right)$ starts to contribute to $|S|$ as the wave packet propagates away from the equator. The increase in wave amplitude with distance $h$ along the magnetic field line is illustrated schematically in Fig. 1b. As shown in Fig. 3a, the nonlinear growth of the chorus elements gradually subsides as the flux of resonant electrons decreases along the field line. This is because the magnitude of the resonance velocity given by Eq. (1) increases with increasing $h$.

\section{Acceleration of relativistic electrons}

In the process of chorus wave generation, untrapped resonant electrons lose energy to the wave, while trapped resonant electrons gain energy. The number of trapped resonant electrons is generally much smaller than that of the untrapped electrons. This causes nonlinear growth of the whistler-mode wave packet as it propagates away from the equator. When $S=-0.4$ the nonlinear growth maximizes due to the effect of the untrapped resonant electrons (Omura et al., 2008), while the trapped resonant electrons undergo an increase in perpendicular velocity and are effectively accelerated. Special trajectories exist for which electrons of several-hundred-keV energy, moving toward the equator, change their direction of motion along the magnetic field and, during their turning motion, are accelerated to $\mathrm{MeV}$ energies in a single interaction. The interaction requires a sufficiently long whistler-mode wave packet and wave amplitudes of the order $\sim 100 \mathrm{pT}$ (Omura et al., 2007; Katoh and Omura, 2007b). The acceleration can take place during the turning motion within a second. This very efficient acceleration mechanism is called relativistic turning acceleration (RTA); see the illustration in Fig. 1d (left). The resonance velocity $V_{R}$ calculated from the cyclotron resonance condition (1) is $V_{R}=\left(\omega-\Omega_{e} / \gamma\right) / k$. For an electron of a few hundred keV such that $\gamma<\Omega_{e} / \omega$, the resonance velocity $V_{R}$ is negative. As the electrons are accelerated by the wave trapping, $\gamma$ increases. When $\gamma=\Omega_{e} / \omega$, $V_{R}$ vanishes. For a larger energy $\gamma>\Omega_{e} / \omega, V_{R}$ becomes positive. The high efficiency of the acceleration process is due to the extended interaction time over the course of the turning motion during which $V_{R} \sim 0$. RTA is very efficient for a long wave packet with a constant amplitude and frequency. For chorus, however, the frequency is time dependent, and the duration of each chorus element is short, of the order of $100 \mathrm{~ms}$. In the simulation of chorus generation, the RTA process takes place in a piecewise manner by different chorus elements, as shown in Fig. 4. The piecewise nature of the interactions with successive chorus elements works favorably for the trapping of the resonant electrons. The wave front of each chorus element can channel a substantial number of resonant electrons into the wave potential well due to the sudden change in the wave amplitude.

After a trapped electron is accelerated by the RTA process and then becomes detrapped, it undergoes adiabatic motion at a constant energy. The resonant electron velocity is positive since the Lorentz factor satisfies the condition $\gamma>\Omega_{e} / \omega$. If the electron now encounters a whistler-mode wave packet near the equator, it can again become trapped and undergo further acceleration, as illustrated in Fig. 1d (right). Acceleration by nonlinear wave trapping is therefore possible even in a very high energy range. The acceleration process corresponding to $\gamma>\Omega_{e} / \omega$ (or to electrons with kinetic energy $\left.E /\left(m_{0} c^{2}\right)>\Omega_{e} / \omega-1\right)$ has been analyzed theoretically, and has been termed ultra-relativistic acceleration (URA) (Summers and Omura, 2007). URA has the important characteristic that its interaction time can be much longer than for the RTA process, even with a short-lived wave packet like a chorus element. If the resonance velocity approaches the group velocity of the whistler-mode wave packet, URA becomes extremely efficient. Figure 4 shows that URA takes place effectively for electrons with energies greater than $2 \mathrm{MeV}$. 
In Fig. 3b, we plot the distribution of energized electrons produced in the chorus generation simulation as a function of energy and position at $t=10000 \Omega_{e}^{-1}$. The colors indicate increments in kinetic energy over the time interval, $10000 \Omega_{e}^{-1}-15000 \Omega_{e}^{-1}$. Electron acceleration in the energy range $0.5-2.0 \mathrm{MeV}$ is due to RTA, while that beyond $2.0 \mathrm{MeV}$ is due to URA. Many particles are energized by $10-40 \mathrm{keV}$ within the time period $5000 \Omega_{e}^{-1}$, i.e. $60 \mathrm{~ms}$. Both RTA and URA are especially effective in slightly offequatorial regions. Here, the chorus wave elements grow to a relatively large amplitude as a result of the nonlinear trapping mechanism.

Energization of radiation belt electrons by gyroresonant interaction with chorus waves has hitherto mainly been treated by quasi-linear diffusion theory. Quasi-linear theory assumes incoherent wideband whistler-mode waves and hence cannot treat nonlinear trapping of resonant electrons by coherent waves. Timescales for electron acceleration due to nonlinear trapping by chorus waves can be much shorter than the corresponding timescales predicted by quasi-linear diffusion theory. Recently it has been demonstrated that both RTA and URA contribute to the formation of a high-energy tail in the electron distribution function in a time-scale of about one hour in the outer radiation belt (Furuya et al., 2008). Nonlinear trapping of radiation belt electrons by coherent whistler-mode waves is emerging as a new paradigm in radiation belt physics.

Acknowledgements. Computation in the present study was performed with the KDK system of RISH and ACCMS (Academic Center for Computing and Media Studies) at Kyoto University. This work was partially supported by Grant-in-Aid 20340135 and 17 GS0208 for Creative Scientific Research "The Basic Study of Space Weather Prediction" of the Ministry of Education, Science, Sports and Culture of Japan. Y. K. is supported by a research fellowship of the Japan Society for Promotion of Science for Young Scientists. D. S. acknowledges support from the Natural Sciences and Engineering Research Council of Canada under grant A-0621.

Topical Editor I. A. Daglis thanks I. Roth for his help in evaluating this paper.

\section{References}

Albert, J. M.: Nonlinear interaction of outer zone electrons with VLF waves, Geophys. Res. Lett., 29(8), 1275, doi:10.1029/2001GL013941, 2002.

Coroniti, F. V., Scarf, F. L., Kennel, C. F., Kurth, W. S., and Gurnett, D. A.: Detection of Jovian whistler mode chorus: Implications for the Io torus aurora, Geophys. Res. Lett., 7(1), 45-48, 1980.

Furuya, N., Omura, Y., and Summers, D.: Relativistic turning acceleration of radiation belt electrons by whistler mode chorus, J. Geophys. Res., 113, A04224, doi:10.1029/2007JA012478, 2008.

Gurnett, D. A., Kurth, W. S., and Scarf, F. L.: Plasma waves near Saturn: Initial results from Voyager 1, Science, 212, 235-239, 1981.
Gurnett, D. A., Kurth, W. S., Scarf, F. L., and Poynter, R. L.: First plasma wave observations at Uranus, Science, 233, 106-109, 1986.

Hospodarsky, G. B., Averkamp, T. F., Kurth, W. S., Gurnett, D. A., Menietti, J. D., Santolik, O., and Dougherty, M. K.: Observations of chorus at Saturn using the Cassini radio and plasma wave instrument, J. Geophys. Res., doi:10.1029/2008JA013237, in press, 2008.

Katoh, Y. and Omura, Y.: Computer simulation of chorus wave generation in the Earth's inner magnetosphere, Geophys. Res. Lett., 34, L03102, doi:10.1029/2006GL028594, 2007a.

Katoh, Y. and Omura, Y.: Relativistic particle acceleration in the process of whistler-mode chorus wave generation, Geophys. Res. Lett., 34, L13102, doi:10.1029/2007GL029758, 2007 b.

Kennel, C. F. and Petschek, H. E.: Limit of stably trapped particle fluxes, J. Geophys. Res., 71(1), 1-28, 1966.

Miyoshi, Y., Morioka, A., Obara, T., Misawa, H., Nagai, T., and Kasahara, Y.: Rebuilding process of the outer radiation belt during the 3 November 1993 magnetic storm: NOAA and Exos-D observations, J. Geophys. Res., 108(A1), 1004, doi:10.1029/2001JA007542, 2003.

Meredith, N. P., Horne, R. B., and Anderson, R. R.: Substorm dependence of chorus amplitudes: Implications for the acceleration of electrons to relativistic energies, J. Geophys. Res., 106, 13 165-13 178, 2001.

Meredith, N. P., Cain, M., Horne, R. B., Thorne, R. M., Summers, D., and Anderson, R. R.: Evidence for chorus-driven electron acceleration to relativistic energies from a survey of geomagnetically disturbed periods, J. Geophys. Res., 108(A6), 1248, doi:10.1029/2002JA009764, 2003.

Omura, Y. and Summers, D.: Dynamics of high-energy electrons interacting with whistler mode chorus emissions in the magnetosphere, J. Geophys. Res., 111, A09222, doi:10.1029/2006JA011600, 2006.

Omura, Y., Furuya, N., and Summers, D.: Relativistic turning acceleration of resonant electrons by coherent whistler mode waves in a dipole magnetic field, J. Geophys. Res., 112, A06236, doi:10.1029/2006JA012243, 2007.

Omura, Y., Katoh, Y., and Summers, D.: Theory and simulation of the generation of whistler-mode chorus, J. Geophys. Res., 113, A04223, doi:10.1029/2007JA012622, 2008.

Roth, I., Temerin, M., and Hudson, M. K.: Resonant enhancement of relativistic electron fluxes during geomagnetically active periods, Ann. Geophys., 17, 631-638, 1999, http://www.ann-geophys.net/17/631/1999/.

Santolik, O., Gurnett, D. A., Pickett, J. S., Parrot, M., and Cornilleau-Wehrlin, N.: A microscopic and nanoscopic view of storm-time chorus on 31 March 2001, Geophys. Res. Lett., 31, L02801, doi:10.1029/2003GL018757, 2004.

Scarf, F. L., Gurnett, D. A., and Kurth, W. S.: Jupiter plasma wave observations: An initial Voyager 1 overview, Science, 204, 991995, 1979.

Smith, A. J., Meredith, N. P., and O'Brien, T. P.: Differences in ground-observed chorus in geomagnetic storms with and without enhanced relativistic electron fluxes, J. Geophys. Res., 109, A11204, doi:10.1029/2004JA010491, 2004.

Summers, D., Thorne, R. M., and Xiao, F.: Relativistic theory of wave-particle resonant diffusion with application to electron acceleration in the magnetosphere, J. Geophys. Res., 103, $20487-$ 
$20500,1998$.

Summers, D. and Ma, C.: A model for generating relativistic electrons in the Earth's inner magnetosphere based on gyroresonant wave-particle interactions, J. Geophys. Res., 105, 2625-2639, 2000.

Summers, D., Ma, C., Meredith, N. P., Horne, R. B., Throne, R. M., Heynderickx, D., and Anderson, R. R.: Model of the energization of outer-zone electrons by whistler-mode chorus during the October 9, 1990 geomagnetic storm, Geophys. Res. Lett., 29(24), 2174, doi:10.1029/2002GL016039, 2002.
Summers, D. and Omura, Y.: Ultra-relativistic acceleration of electrons in planetary magnetospheres, Geophys. Res. Lett., 34, L24205, doi:10.1029/2007GL032226, 2007.

Tsurutani, B. T. and Smith, E. J.: Postmidnight chorus: a substorm phenomenon, J. Geophys. Res., 79, 118-127, 1974.

Varotsou, A., Boscher, D., Bourdarie, S., Horne, R. B., Glauert, S. A., and Meredith, N. P.: Simulation of the outer radiation belt electrons near geosynchronous orbit including both radial diffusion and resonant interaction with whistler-mode chorus waves, Geophys. Res. Lett., 32, L19106, doi:10.1029/2005GL023282, 2005. 